\title{
THE MOLECULAR OUTFLOW AND CO BULLETS IN HH111
}

\section{J. CERNICHARO}

Instituto de Estructura de la Materia, Dpto Física Molecular Serrano 123, 28006 Madrid, Spain

R. NERI

IRAM, Domaine Universitaire, 300 rue de la Piscine 38406 St Martin d'Hères, France

AND

BO REIPURTH

European Southern Observatory

Casilla 19001, Santiago 19, Chile

\begin{abstract}
We present high angular resolution observations of the molecular outflow associated with the optical jet and $\mathrm{HH}$ objects of the HH111 system. Interferometric observations in the $\mathrm{CO} J=2-1$ and $\mathrm{J}=1-0$ lines of the high velocity bullets associated with $\mathrm{HH} 111$ are presented for the first time. The molecular gas in these high velocity clumps has a moderate kinetic temperature and a mass of a few $10^{-4} \mathrm{M}_{\odot}$ per bullet. We favor the view that $\mathrm{HH}$ jets and $\mathrm{CO}$ bullets, which represent different manifestations of the same physical phenomena, are driving the low-velocity molecular outflow.
\end{abstract}

\section{Introduction}

The often deeply embedded stars which drive Herbig-Haro (HH) flows are, as a group, considered among the youngest stars known. The supersonic shocked jets which emanate from these stars testify to the current release of large amounts of energy in or near the nascent stars. In the first steps of star formation, orbital angular momentum of the collapsing material has to be evacuated so that matter continues to be accreted on to the protostar. This can happen by ejecting a fraction of the accreting material - which takes away most of the angular momentum - in a wind of magnetic 


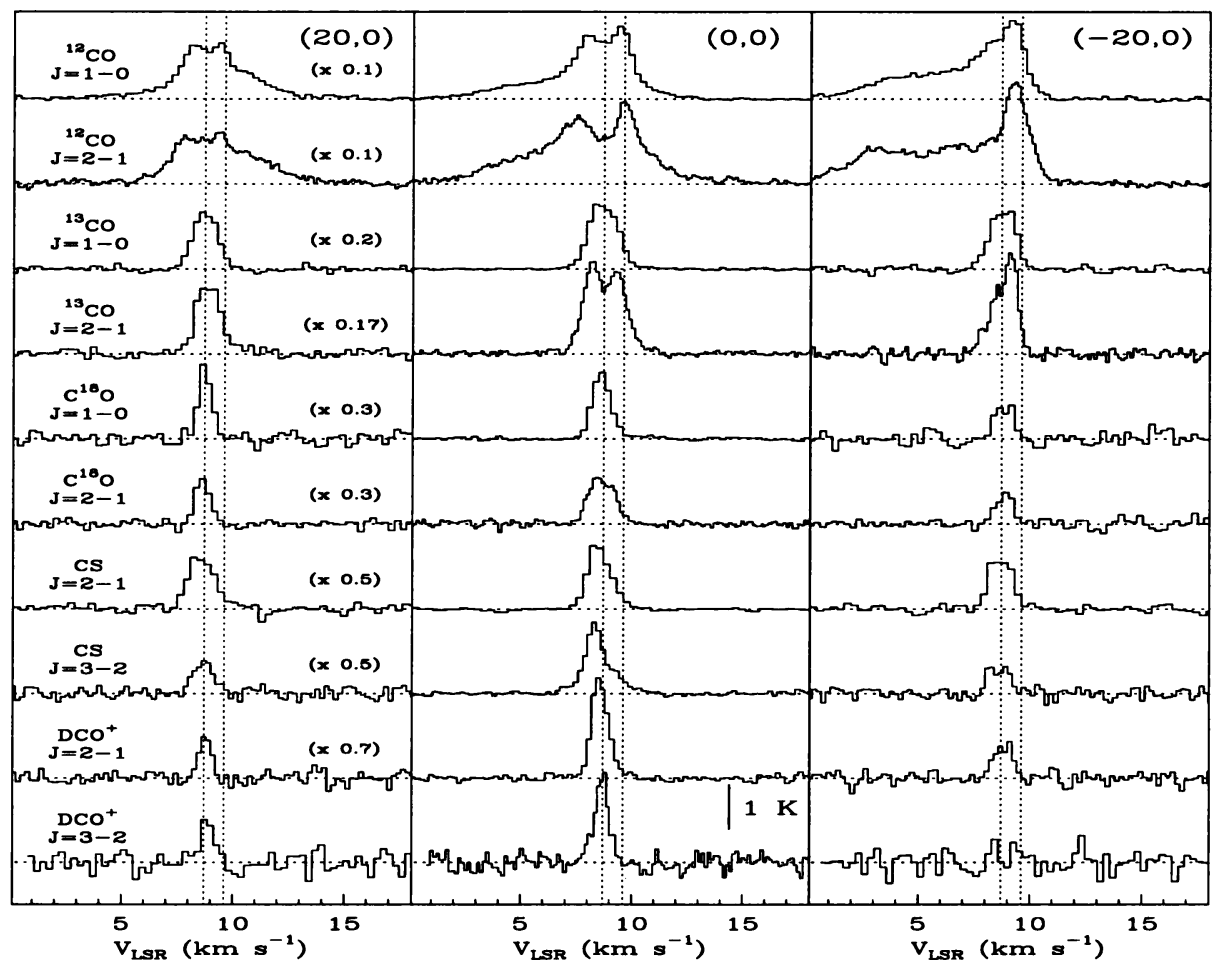

Figure 1. Molecular line emission of $\mathrm{CO},{ }^{13} \mathrm{CO}, \mathrm{C}^{18} \mathrm{O}, \mathrm{CS}$ and $\mathrm{DCO}^{+}$toward selected positions along the optical jet of HH111 (indicated in arcsec at the top-right of each upper panel). The central position corresponds to the VLA source $\left(\alpha_{1950}=05^{h} 49^{m} 9.3^{s}\right.$, $\left.\delta_{1950)}=2^{\circ} 47^{\prime} 48^{\prime \prime}\right)$. The high velocity gas is particularly prominent over the blue part of the jet.

origin (Shu et al 1987; Pelletier \& Pudritz 1992; see also the papers by Shu and Pudritz in this volume) with velocities as high as $10^{3} \mathrm{~km} \mathrm{~s}^{-1}$. The wind interacts with the ambient gas producing shocks that are detected as optical jets and HH objects (Reipurth 1989; Reipurth and Cernicharo 1995; Ray et al 1996; see also the reviews by Reipurth and Bally in this volume).

The HH jets seem to drive the bipolar molecular outflows (Cernicharo \& Reipurth 1996; hereafter CR96) detected around protostars, a second massloss driven phenomenon taking place during the earliest evolutionary stages of the star formation process (Bachiller, 1996). A particular nice example of optical and molecular jets can be found in HH111. This HH system, with its high velocity $\mathrm{CO}$ bullets and its highly collimated molecular outflow, is the ideal object to test the different models proposed to explain how molecular outflows are driven (CR96). We present in this paper a description of the molecular gas at low and extremely high velocity gas around the HH111 system. 


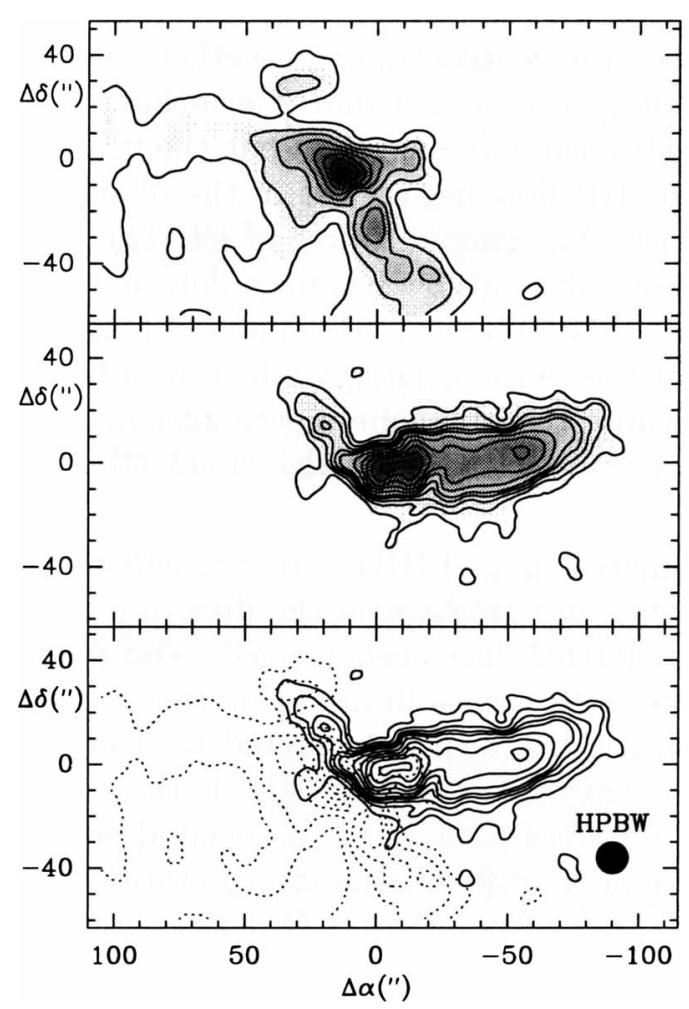

Figure 2. The two upper panels show integrated $\mathrm{CO}$ intensity for velocities between -5 and $+7 \mathrm{kms}^{-1}$ and between +10.5 and $+15 \mathrm{kms}^{-1}$, respectively. The beam-width is 12 " and the spacing of the data over the full region is 7". The third panel combines the blue and redshifted lobes. An optical picture of HH111 can be seen in Reipurth \& Heathcote (this volume).

\section{The HH111 system}

The HH111 optical jet is located in the L1617 cloud in the Orion B cloud complex and was discovered by Reipurth (1989). The HH complex stretches over 367", corresponding, for a distance distance of $460 \mathrm{pc}$, to $0.82 \mathrm{pc}$. It consists of a bright highly collimated jet, a small faint counter-jet and at least 4 bow shocks, two on each side of the source. Additional very distant bow shocks on either side of the central source have also been detected by Reipurth, Bally \& Devine (1997) (see also the review by Bally \& Devine in this volume). The proper motions along the jet are large, between 300 and $600 \mathrm{kms}^{-1}$ and directed away from the driving source, IRAS 0591+0247, which is a deeply embedded $25 \mathrm{~L}_{\odot}$ source (Reipurth et al, 1992). The source has been detected at 2.0 and $3.6 \mathrm{~cm}$ at the VLA (Rodriguez \& Reipurth, 1994). It has also been detected in the continuum at $3 \mathrm{~mm}$ by Stapelfeldt 
\& Scoville (1993) and Yang et al (1997). We have recently detected the continuum emission from the central source, together with extended emission from the surrounding core, at $1.3 \mathrm{~mm}$ (Cernicharo et al 1997). The observed flux $(\simeq 450 \mathrm{mJy})$ strongly suggests that the central source could be a class 0 source. The HH flow lies almost in the plane of the sky, with an inclination of only $10^{\circ}$. The energy source of $\mathrm{HH} 111$ is embedded in a $30 \mathrm{M}_{\odot}$ cloud core. A molecular outflow has been detected along the axis of the HH complex (Reipurth \& Olberg, 1991). The red (eastern) lobe has an extension of $0.7 \mathrm{pc}$, which is twice as large as the blue lobe. This difference probably originates because the blue lobe moves into almost empty space, whereas the red lobe is interacting with the cloud (Reipurth \& Olberg (1991); CR96).

The ${ }^{13} \mathrm{CO}$ interferometric map of HH111 by Stapelfeldt and Scoville indicates a North-South velocity gradient in the direction of the core where the powering source of HH111 has been formed. The recent CS map of Yang et al (1997) taken with the Nobeyama interferometer also suggests such a velocity gradient. It has been interpreted by Yang et al (1997) as infalling gas around the exciting source of HH111. However, the fact that the emission from the CS lines show wings associated with the molecular outflow (see Figure 1) renders difficult the interpretation of the data. The presence of a well defined cavity in the ${ }^{12} \mathrm{CO} \mathrm{J}=2-1$ emission as observed with the 30-m IRAM telescope (CR96) and in the ${ }^{12} \mathrm{CO} \mathrm{J}=1-0$ interferometric maps of Cernicharo et al (1997; see below) and Nagar et al (1997) (see also Reipurth \& Cernicharo 1995), indicates that the kinematics of the gas around the central source is affected by several different physical processes.

The emission of ${ }^{12} \mathrm{CO},{ }^{13} \mathrm{CO}$ and $\mathrm{C}^{18} \mathrm{O}$ in the $\mathrm{J}=2-1$ and $\mathrm{J}=1-0$ lines, together with that of the $\mathrm{J}=2-1$ and $\mathrm{J}=3-2$ lines of $\mathrm{CS}$ and $\mathrm{DCO}^{+}$, is shown in Figure 1 at selected positions along the optical jet of HH111 (CR96, and unpublished data). The line wings are detected even in the emission of the rotational transitions $\mathrm{J}=2-1$ and $\mathrm{J}=3-2$ of $\mathrm{CS}$. The molecular hydrogen density in the core is as large as $10^{5} \mathrm{~cm}^{-3}$. The ${ }^{12} \mathrm{CO} \mathrm{J}=2-1$ and $\mathrm{J}=1-0$ emission reaches its maximum at a velocity of $9.7 \mathrm{kms}^{-1}$. The gas at this velocity extends over the blue part of the jet. It has its emission maxima at positions -10 to -20 arcsec from the exciting source of the HH111 system (Fig.1) and has been interpreted by CR96 as the result of the interaction of the jet with the ambient gas, constituting the rear part of an expanding cavity around the optical jet. The gas kinematics is similar to that proposed by Martín-Pintado \& Cernicharo (1987) for HH12 (see also Cernicharo 1991).

In the direction of the core some molecular lines show signs of selfabsorption (CS $\mathrm{J}=2-1$ and $\mathrm{J}=3-2,{ }^{13} \mathrm{CO} \mathrm{J}=2-1$ and $\mathrm{C}^{18} \mathrm{O} \mathrm{J}=2-1$ ). However, this double peaked line profile (see Figure 1) is probably due to the kine- 


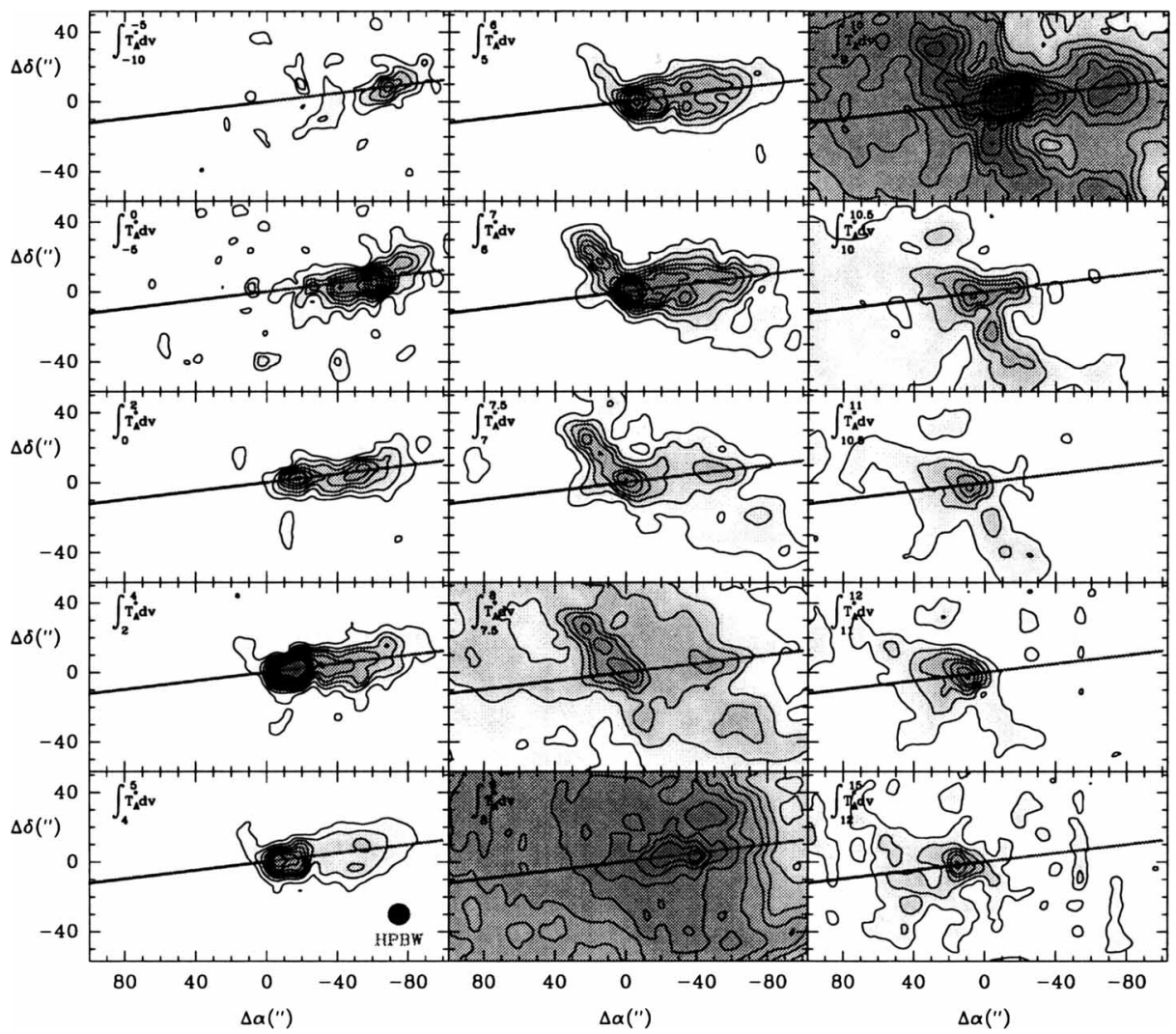

Figure 3. Integrated $\mathrm{CO} \mathrm{J}=2-1$ intensity for the velocity intervals indicated at the top-left of each panel. The thick line crossing the different panels indicates the axis of the $\mathrm{HH}$ flow. The first feature that appears in this figure is the long molecular jet coinciding with the $\mathrm{HH}$ flow, see panels corresponding to velocities lower than $+2 \mathrm{kms}^{-1}$. At velocities between +5 and $+7.5 \mathrm{kms}^{-1}$ a second jet is clearly visible running from the VLA source towards the Northeast. This jet has its red counterpart running from the VLA source towards the Southwest at velocities between +10 and $+12 \mathrm{kms}^{-1}$. The red counterpart of the main blue molecular jet also appears at these velocities but it is much less collimated and is oriented in the direction traced by the optical jet. The ambient gas appears at velocities between +7.5 and $+10 \mathrm{kms}^{-1}$ (adapted from data shown in CR96)

matics of the gas in the expanding cavity mentioned above.

\section{The low velocity outflow}

The short blue lobe found by Reipurth \& Olberg (1991) is seen in the higher resolution CO J=2-1 data of CR96 to be a highly collimated molecular jet about 90 arcsec long with a collimation factor of about 9 (see Figures 2, 3 , and 4 and CR96). The blue $\mathrm{CO}$ gas lobe ends at the bow shock $\mathrm{P}$ and the optical knots Q,R,S (see Reipurth 1989). The CO blue lobe is shorter 
than the $\mathrm{HH}$ lobe, in which the most distant bow shock $\mathrm{V}$ is located almost 150 arcsec from the VLA source. At these larger distances the quiescent gas of the cloud has a low visual extinction and volume density (CR96). Hence, the $\mathrm{HH}$ jet is emerging into a low density medium and escaping the cloud. CR96 suggest that the molecular outflow is formed by material dragged along from inside the globule rather than gas that has been swept up from the thin ambient medium and compressed. As shown in the infrared images of Gredel \& Reipurth $(1993,1994)$ there is a second bipolar jet, HH121, emanating from the VLA source. In Figure 3 the quadrupole flow is particularly apparent (see also Figure 2 and CR96). The presence of two outflows with nearly spatially coinciding powering sources makes it difficult to develop any simple model of the kinematics of the gas around the VLA source (see also above).

Although the blue and red integrated intensity maps shown in Figure 2 seem to indicate a rather smooth appearance for the HH111 molecular jet, the velocity interval maps of Figure 3 and the velocity-position diagram shown by CR96 reveal that there are several clumps in the outflow. In particular, the spatial distribution of the gas at velocities between +4 and $+6 \mathrm{kms}^{-1}$, and at distances larger than $20^{\prime \prime}$ West, seem to delineate the walls of an expanding cavity or tube surrounding the optical jet. The front and the rear of such a cavity could be traced by the gas at velocities between +7 and $+10 \mathrm{kms}^{-1}$ (rear) and between 0 and $+4 \mathrm{kms}^{-1}$ (front), see also Cabrit \& Bertout (1990). The same spatial structure, i.e. an expanding tube or cavity, could also fit the kinematics of the gas near the VLA source as indicated in Figures 3 and 4.

The maps in Figure 2 and 3, and those presented in CR96, were taken with a full sampling in the $\mathrm{J}=2-1$ line of ${ }^{12} \mathrm{CO}$ (HPBW=12"). Recently, Nagar et al (1997) have shown an interferometric map of the emission of the $\mathrm{J}=1-0$ line of ${ }^{12} \mathrm{CO}$. The angular resolution of their observations (5.5" $\mathrm{x} 6$ ") allow them to resolve the structure of the low velocity gas around the optical jet. They found, like CR96, a hollow tubular structure around the jet. CR96 also reported the presence of different clumps associated with the cavity. Some prelimary results of our PdBI CO J=1-0 interferometric maps were presented by Reipurth \& Cernicharo (1995). Figure 4 shows our final interferometric map from a mosaic covering 5 fields along the blue part of the jet (Cernicharo et al 1997). The uv coverage, sensitivity and spatial resolution (3" $\times 3.5 ")$ are better than those of Nagar et al (1997) and, in addition to the cavity reported by CR96 and Nagar et al, confirm the presence of several clumps in the emission of $\mathrm{CO}$ at low velocity. They are located along the optical jet and are associated with some of the $\mathrm{HH}$ objects. In figure 4 we can easily see the rear $\left(\mathrm{v}=9\right.$ to $\left.10 \mathrm{kms}^{-1}\right)$ and front $\left(\mathrm{v} \simeq 3-5 \mathrm{kms}^{-1}\right)$ parts of the cavity. The walls of the cavity are seen at 
velocities between 5.8 and $7.2 \mathrm{kms}^{-1}$, being particularly prominent at 6.4 $\mathrm{kms}^{-1}$. The red counterpart of the HH111 molecular outflow appears, like in the single dish maps of Figure 3, much less collimated. In addition, the CO blue emission shows two clumps (bow shocks ?) at positions 25"-35" West of the VLA source $\left(\mathrm{v}=7.2-8.1 \mathrm{kms}^{-1}\right.$ in Figure 4$)$ and 60 " West of the VLA source $\left(\mathrm{v}=9.3-9.5\right.$ and $\left.6.4 \mathrm{kms}^{-1}\right)$. These clumps are also detected in the single dish maps of CR96 (see their Figure 3). The HH121 flow is also seen in Figure 4.

\section{The high velocity gas along the Herbig-Haro jet}

CR96 obtained a CO spectrum of the inner part of the HH jet by averaging all the ${ }^{12} \mathrm{CO} \mathrm{J}=2-1$ data between the VLA source and position 60 " West along the jet. The spectrum consists of a broad and weak emission feature centered at $-70 \mathrm{kms}^{-1}$. This weak emission could be associated to the neutral counterpart of the inner HH jet. Why is this high velocity CO emission so weak ? One possibility is that the presence of shocks in the $\mathrm{HH}$ jet has dissociated the $\mathrm{CO}$ in the jet stream. Alternatively, as suggested by CR96, any CO gas in the body of the jet may have so high a kinetic temperature that the opacity in the $\mathrm{J}=1-0$ and $\mathrm{J}=2-1$ line of $\mathrm{CO}$ drops, resulting in the $\mathrm{CO}$ emission being weak. Liseau et al (1996) have detected emission from high-J lines of CO in HH54. They have derived a kinetic temperature for the shocked gas in this $\mathrm{HH}$ object of $330 \mathrm{~K}$. With such a high value for $\mathrm{T}_{K}$, and taking into account the low $\mathrm{CO}$ column densities involved in the high velocity molecular jet, the expected intensities for the $\mathrm{J}=2-1$ and $\mathrm{J}=1-0$ lines of $\mathrm{CO}$ could be very low. By assuming a kinetic temperature of $100 \mathrm{~K}$, a $\mathrm{CO}$ abundance of $10^{-4}$, a distance of 460 $\mathrm{pc}$, thermalization for the $\mathrm{J}=2-1 \mathrm{CO}$ line, and the $\mathrm{CO} / \mathrm{H}_{2}$ conversion factor of Cernicharo \& Guélin (1987), CR96 derived a mass for this high velocity neutral gas of $0.0007 \mathrm{M}_{\odot}$; the associated momentum is $\simeq 0.3 \mathrm{M}_{\odot} \mathrm{kms}^{-1}$.

\section{The high velocity gas at the end of the Herbig-Haro jet}

The gas associated with the low velocity molecular outflow in the HH111 system covers the region where the optical jet has been detected (see figure 2 and CR96) and ranges from -10 to $+15 \mathrm{kms}^{-1}$. However, just beyond bow shock $\mathrm{P}$ and optical knots $\mathrm{Q}, \mathrm{R}$ and $\mathrm{S}$ (see Reipurth 1989), where no more outflow emission could be expected, CR96 found strong CO emission at extremely high velocity (EHV).

Beyond the optical bow shock $\mathrm{P}$, which is at position 75 " from the VLA source, the CO emission appears at velocities between -40 and -80 $\mathrm{kms}^{-1}$ (see figure 5 of CR96). Assuming an angle of $10^{\circ}$ to the plane of the sky for the HH 111 complex as derived from radial velocity and proper 


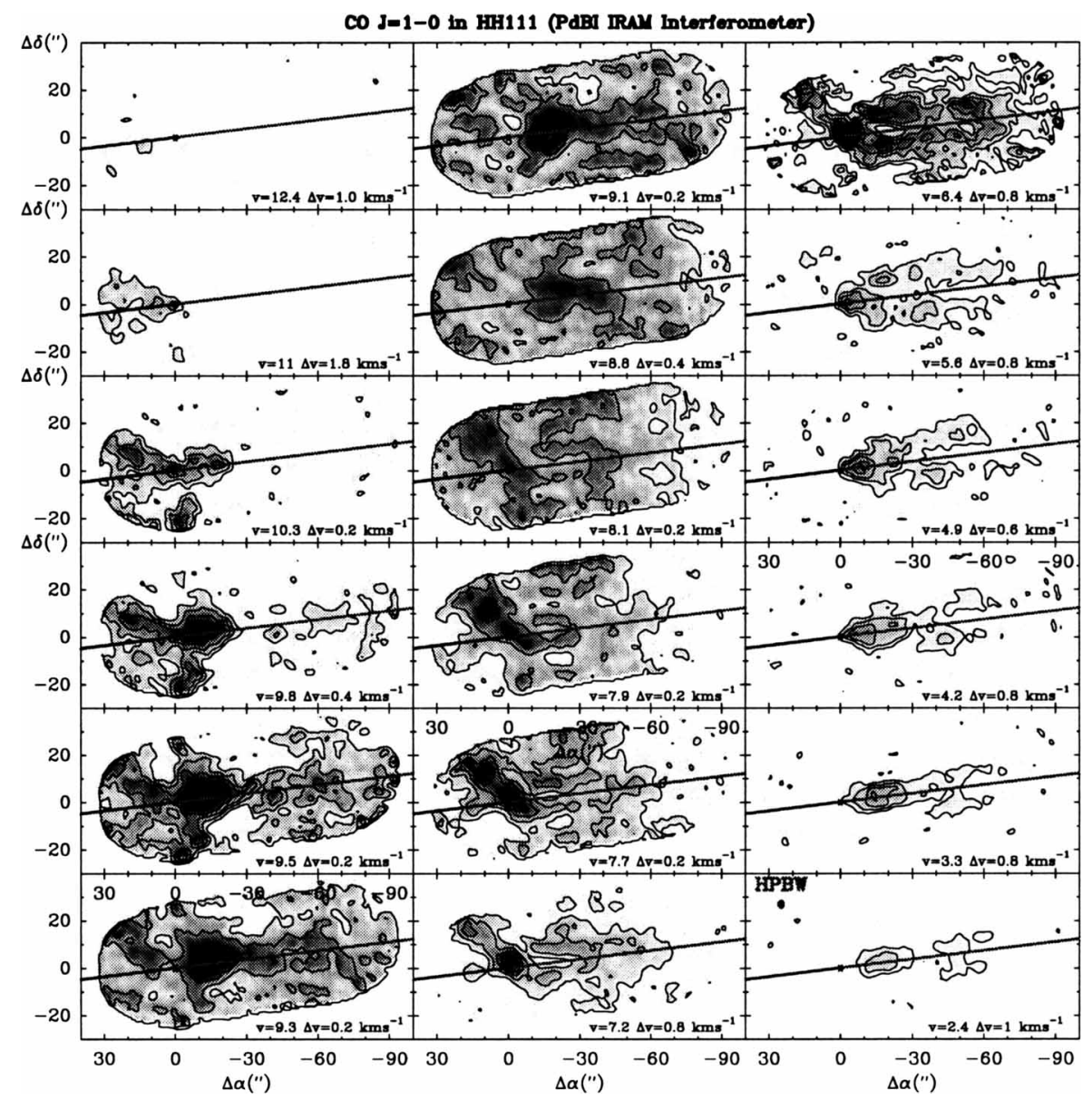

Figure 4. Interferometric maps of the $\mathrm{J}=1-0 \mathrm{CO}$ emission taken with the PdBI. The maps result from a mosaic of 5 overlapping fields covering 120 ". The HPBW is 2.5 " $\times 3.5$ " and is indicated in the lower-right panel. Zero spacing data from the $30-\mathrm{m}$ has been merged with the PdBI data. The thick line in each panel indicates the orientation of the HH111 optical jet.

motion measurements of the $\mathrm{HH}$ jet (Reipurth et al, 1992), the true space velocity of the high velocity $\mathrm{CO}$ emission is between 250 and $500 \mathrm{kms}^{-1}$. This gas is detected extending from 80 " to 150 " West of the VLA source. The first EHV CO emission appears just after knot $\mathrm{P}$ at a LSR velocity of $-60 \mathrm{kms}^{-1}$ which is identical to the LSR velocity of the ionized gas in this knot (Reipurth, 1989). The EHV CO emission consists of four bullets at positions 80" (knot P), 110" , 125" and 150" (knot V) West (see Figures 5 and 6 of CR96). Like for the low velocity gas at these positions, the velocity dispersion in the EHV bullets is very large as it could be expected from the interaction of the EHV gas with the ambient gas (see above). The EHV 
${ }^{12} \mathrm{CO}(2-1)$ along the HH111 jet

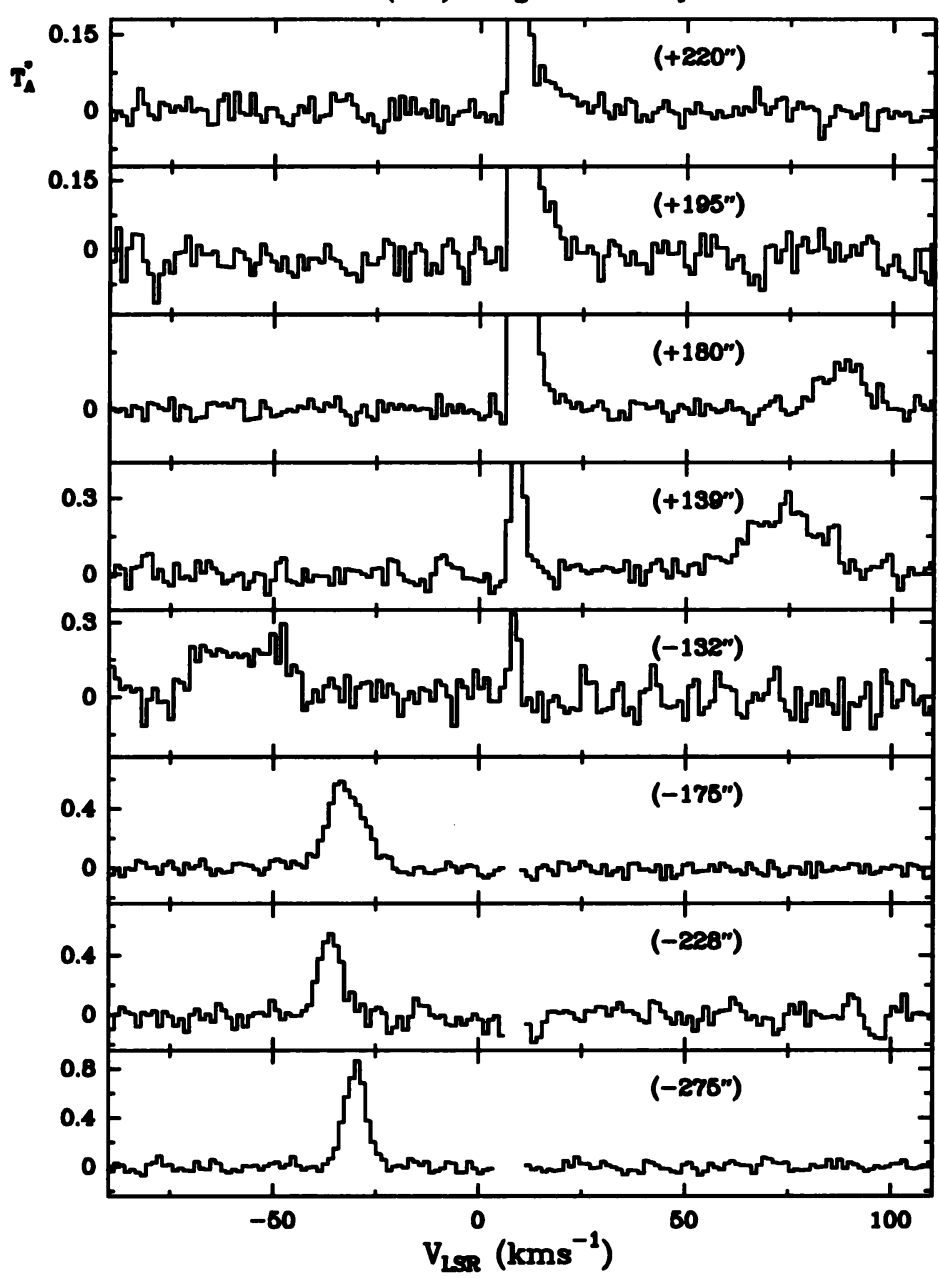

Figure 5. ${ }^{12} \mathrm{CO} \mathrm{J}=2-1$ spectra at selected positions along the axis of the HH111 jet. The $\mathrm{CO}$ bullets and the $\mathrm{CO}$ high velocity gas were discovered first in the blue part of the jet (Cernicharo \& Reipurth, 1996). The redshifted high velocity gas (upper 4 panels) has also been observed at positions nearly symmetric to that of the blue high velocity CO gas, lower 3 panels (Cernicharo et al, 1997)

CO gas disappears beyond knot V. This optical knot has an LSR velocity of $-70 \mathrm{kms}^{-1}$ (Reipurth 1989), which is also identical to the velocity of the neutral gas at this position.

The red counterpart of the blue high velocity gas has been recently detected by Cernicharo et al (1997) -see Figure 5 . The red high velocity gas appears at positions nearly symmetric to those of the blue components. It is barely resolved in the direction of the jet at position +139 " (symmetric to 
knot V) and also shows a very broad velocity dispersion. Further away, at +180 " from the VLA source, another red high velocity emission is detected. But this time, like for the high velocity blue bullets of CR96 (see below), the emission is unresolved by the 12" beam of the 30-m IRAM telescope and could be the red counterpart of Bullet 1 described below.

\section{The high velocity gas further away : the CO bullets}

Beyond the optical bow shock V, CR96 discovered three CO bullets (hereafter refered to as Bullet 1, 2 and 3, in order of increasing distance from the source) with separations of $\simeq 55$ ". No optical emission is associated with these bullets. However, they are perfectly aligned along the direction defined by the optical jet. The Bullet 3 appears at $0.63 \mathrm{pc}$ from the VLA source (position 280" West). The de-projected velocity of the three bullets is about $240 \mathrm{kms}^{-1}$. This velocity implies an episodic ejection of matter every $\simeq 500$ years. The outermost bullet could be emitted from the central source some 2600 years ago. Note that the distance between Bullet 1 and that associated with the optical bow shock $\mathrm{P}$ is also about 55 ". The bright bow shock V, however, is located midway between knot $\mathrm{P}$ and Bullet 1 . From the maps shown in Figure 5 of CR96 the bullets appear to be barely resolved with the 12 " beam at $230 \mathrm{GHz}$. Recently, Cernicharo et al (1997) have made high angular resolution maps with the PdBI interferometer in the $\mathrm{J}=1-0$ and $\mathrm{J}=2-1$ lines of CO. Figure 6 shows the resulting spatial distribution of the CO $\mathrm{J}=1-0$ emission. The $\mathrm{J}=2-1$ line looks very similar and the emission of both lines is resolved by the resulting beams. The $\mathrm{CO}$ $\mathrm{J}=2-1 / \mathrm{J}=1-0$ intensity ratio in the three bullets is about 1.5 (see also CR96). In the optically thin case and assuming equal excitation conditions for the $\mathrm{J}=1-0$ and $\mathrm{J}=2-1$ lines, this ratio could correspond to excitation temperatures of about $12-20 \mathrm{~K}$. Assuming optically thin emission and $\mathrm{T}_{e x}=15$ $\mathrm{K}$, then the masses of the bullets are a few times $10^{-4}$ solar masses, i.e. similar to that of the bullets in other young low mass star forming regions (see Bachiller et al. 1990; Bachiller \& Cernicharo 1990).

\section{Molecular outflows and Herbig-Haro jets}

Since $\mathrm{HH}$ jets are episodic, with dynamical ages of the order of $10^{3} \mathrm{yr}$, the longer lasting molecular outflows would be the time averaged result of the momentum transferred from the many pulses of $\mathrm{HH}$ jets passing through their ambient medium.

Based on line ratios and shock models, Hartigan, Morse, Raymond (1995) derive a mass loss rate of $1.8 \times 10^{-7} \mathrm{M}_{\odot} \mathrm{yr}^{-1}$ for the HH 111 jet. CR96 derived a time averaged mass transport rate for the CO bullets of $4 \times 10^{-7} \mathrm{M}_{\odot} \mathrm{yr}^{-1}$ Given the uncertainties involved, the mass loss de- 

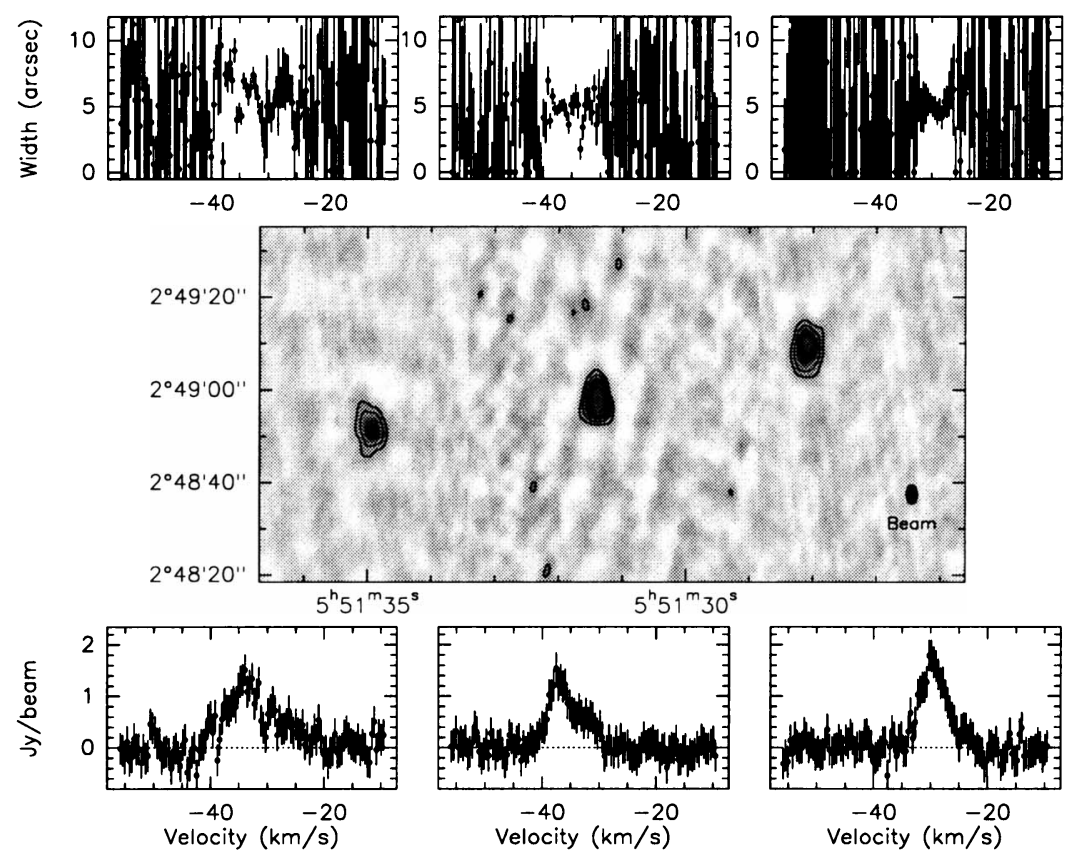

Figure 6. Maps of ${ }^{12} \mathrm{CO} \mathrm{J}=1-0$ taken with the IRAM PdBI intereferometer of the three bullets discovered by CR96. The central map show the integrated intensity distribution of the gas in each bullet. The three lower panels show the average CO $\mathrm{J}=1-0$ spectrum in each bullet. The top panels show the spatial size for each velocity channel. Note that the three bullets are resolved with the PdBI interferometer and that they have a typical size of 5 arcsec. There is considerable velocity structure inside each $\mathrm{CO}$ bullet. From Cernicharo et al (1997)

rived from optical and radio observations are identical. It would seem that the similar mass transport rates in the $\mathrm{HH}$ jet and the $\mathrm{CO}$ bullets provide strong support for the view that these $\mathrm{CO}$ bullets are not made of ambient material that have been accelerated to these very high velocities, but are mostly composed of material ejected from the central engine (note also the excellent agreement in velocities at optical and radio wavelengths found by CR96). The mass loss rate quoted above corresponds to a momentum rate of $1.2 \times 10^{-4} \mathrm{M}_{\odot} \mathrm{yr}^{-1} \mathrm{kms}^{-1}$. The momentum in the molecular gas at intermediate velocities is about $1 \mathrm{M}_{\odot} \mathrm{kms}^{-1}$. If the jet momentum were transfered to the ambient gas with $100 \%$ efficiency, the jet would have to maintain on average the same level of activity for $8 \times 10^{3} \mathrm{yr}$. In this time span, the VLA source would have roughly 20 ejections of matter, if the frequency is one every $500 \mathrm{yr}$. The momentum in each bullet is typically $0.05 \mathrm{M}_{\odot} \mathrm{kms}^{-1}$. Consequently, this number of bullets could account for the 
momentum in the intermediate velocity gas if it was transfered with $100 \%$ efficiency. The fact that the outermost bullets have very high velocities tells us that the momentum transfer cannot be $100 \%$ efficient, and consequently the $\mathrm{HH}$ jet could be driving the observed molecular outflow only if the dynamical age of the complex is larger than we derive. As mentioned earlier, new observations by Reipurth, Bally \& Devine (1997, see also the review by Bally \& Devine in this volume) have shown that the HH 111 jet complex is much larger than hitherto thought, so it appears that the $\mathrm{HH}$ jet may indeed be able to drive the molecular outflow, even with a much lower momentum transfer efficiency.

Acknowledgements: J. Cernicharo gratefully acknowledges the support of the Spanish DGES for this research under grant number APC960168 and ESP96-2529-E.

\section{References}

Bachiller, R., 1996, Annu. Rev. Astron. Astrophys., 34, 111

Bachiller R., Cernicharo J., 1990, A\&A, 239, 276

Bachiller R., Cernicharo J., MartinPintado J., Tafalla M., Lazareff B., 1990, A\&A, 231, 174

Cernicharo, J., 1991, in The Physics of Star Formation and Early Stellar Evolution, , pag. 287, Eds. C. Lada \& N.D.Kylafis, NATO ASI Series, Kluwer Academic Publishers

Cernicharo J., Guélin M., 1987, A\&A, 176, 299

Cernicharo, J., Reipurth, B., 1996, ApJ, 460, L57 (CR96)

Cernicharo, J., Neri, R., Reipurth, B., 1997, in preparation

Gredel, R., Reipurth, B., 1993, ApJ, 407, L29

Gredel, R., Reipurth, B., 1994, A\&A, 289, L19

Liseau, R., Ceccarelli, C., Larsson, B., et al, 1996, A\&A, 315, L181

Martín-Pintado, J., Cernicharo, J., 1987, A\&A, 176, L27

Nagar, N.M., Vogel, S.N., Stone, J.M., Ostriker E., 1997, ApJ, in press (see also the poster-book of this conference)

Pelletier G., Pudritz R.E., 1992, Astrophys. J., 394, 117

Ray, T.P., Mundt, R., Dyson J., Falle, S.A.E.G., Raga, A., 1996, ApJ, 468, L103-L106

Reipurth, B., Nature, 1989, 340, 42

Reipurth, B., Olberg, M., 1991, A\&A, 246, 535

Reipurth, B., Raga, A.C., Heathcote, S., 1992, ApJ, 392, 145

Reipurth, B., Cernicharo, J., 1995, Rev. Mex. Astron. Astrophys., (Serie de Conferencias), $1,43,1995$

Reipurth, B., Bally, J., Devine, D., 1997, AJ, submitted

Rodriguez, L.F., Reipurth, B., 1994, A\&A, 281, 882

Shu F. Adams, F.C., Lizano, S., 1987, Annu. Rev. Astron. Astrophys., 25, 23

Stapelfeldt, K.R., Scoville, N.Z., 1993, ApJ, 408, 239

Yang J., Ohashi, N., Yan, J., Liu, C., Kaifu, N., Kimura, H., 1997, ApJ, 475, 683 